\title{
Práticas de higiene bucal de pacientes em hemodiálise
}

\author{
Practices of bucal hygiene of patients in hemodialysis
}

Kellyn de Bastos Silva*

Ricardo Sartori*

\section{Resumo}

Introdução: os hábitos de higiene bucal de pacientes em hemodiálise podem melhorar sua qualidade de vida e possibilitar o sucesso do transplante renal, entretanto, esse é um assunto pouco evidenciado nas clínicas de terapia substitutiva (hemodiálise) atualmente. Objetivo: avaliar a higiene bucal dos pacientes renais crônicos em tratamento de hemodiálise. Materiais e método: trata-se de um estudo observacional transversal analítico, cuja população de estudo foram pacientes com doença renal crônica no serviço de referência para tratamento hemodialítico da $13^{\text {a }}$ Coordenadoria Regional de Saúde, localizado no município de Santa Cruz do Sul, RS. As técnicas utilizadas para coleta dos dados foram a entrevista interpessoal padronizada e exames clínicos intrabucais, juntamente com a análise do histórico médico digital da instituição. Resultados: a amostra teve predomínio de homens $(59,21 \%)$, com faixa etária de 22 a 89 anos; dos 76 pacientes participantes da pesquisa, 39,47\% utilizam prótese total; a média de prevalência do índice de placa visível foi de $54,137 \%$, e a do índice de sangramento gengival de $16,765 \%$. Os resultados do estudo foram analisados por meio de estatística descritiva e testes de hipóteses. O nível de significância utilizado como critério de aceitação ou rejeição nos testes estatísticos foi de $5 \%(p<0,05)$. Conclusão: os pacientes em hemodiálise avaliados possuem um perfil de higiene bucal precário, com alta prevalência de placa e cálculo dental, o que comprova a necessidade de maior atenção odontológica diversificada e integral para conscientização e melhoria da saúde bucal.

Palavras-chave: Assistência odontológica para doentes crônicos. Avaliação de higiene bucal. Insuficiência renal crônica.

\section{Introdução}

No Brasil, em julho de 2016, o número total estimado de pacientes em hemodiálise era de 122.825. Considerando o período de 2013 a 2016, as taxas de incidência e prevalência de pacientes em diálise tenderam a aumentar de forma constante, e a mortalidade ficou estável. A taxa anual de mortalidade bruta foi de $18,2 \%$. Dos pacientes prevalentes, $92 \%$ estavam em hemodiálise; $8 \%$, em diálise peritoneal; e 29.268 (24\%), em fila de espera para transplante. ${ }^{1}$

Pacientes em terapia substitutiva (hemodiálise) possuem várias alterações bucais, as quais são relevantes para o atendimento odontológico. São algumas delas: halitose, maior acúmulo de cálculo, gengivite, periodontite, fluxo salivar diminuído, xerostomia e parotidite. Sabendo disso, é de extrema importância a avaliação da higiene bucal dos pacientes em hemodiálise, não somente para a determinação da prevalência de alterações bucais, mas também para a prevenção na saúde bucal.,3

A doença periodontal pode ser fator de comorbidade e fonte de inflamação e causar ou facilitar o dano renal. Já a insuficiência renal crônica (IRC) predispõe e agrava a doença periodontal. A diminuição dos focos de inflamação melhora a glicemia e o controle metabólico, resultando em maior qualidade de vida, por melhorar a condição sistêmica. ${ }^{4,5}$

$\mathrm{Na}$ literatura, não há número significante de trabalhos que evidenciam a importância da higiene bucal para pacientes em hemodiálise. Estudo realizado por Barros et. al. ${ }^{6}$ (2014) mostrou grande significância, pois discute a deficiência dos hábitos de higiene bucal dos pacientes nefropatas e a necessidade de motivação e orientação do cirurgião-dentista para melhorar a qualidade de vida desses pacientes, possibilitando o sucesso de futuros tratamentos, inclusive, dos transplantes renais. 
A sociedade acadêmico-científica necessita de mais pesquisas nesta área, relevando a necessidade de inserção do cirurgião-dentista em centros clínicos de hemodiálise. Os pacientes carecem de atenção odontológica diversificada e voltada às consequências do tratamento hemodialítico, melhorando a qualidade de vida. Traçar essa condição de higiene bucal estabelece um conceito de tratamento integrado e interdisciplinar, de acordo com as necessidades desses pacientes. ${ }^{7}$

O objetivo principal deste trabalho é avaliar a higiene bucal dos pacientes renais crônicos em tratamento de hemodiálise no serviço de referência para tratamento hemodialítico, no município de Santa Cruz do Sul, questionando sobre seus hábitos comportamentais e de higiene bucal, relacionando a situação de higiene bucal às consequências da hemodiálise, promovendo a conscientização e a melhora da saúde bucal desses pacientes.

\section{Materiais e método}

Esta pesquisa compreende um estudo observacional transversal analítico. ${ }^{8} \mathrm{O}$ material bibliográfico foi selecionado nos idiomas português, inglês e espanhol e a partir das seguintes bases de dados: Lilacs, Bireme, SciELO, Google Acadêmico e PubMed; além do serviço de Comutação Bibliográfica da biblioteca da Universidade de Santa Cruz do Sul. Foram considerados os materiais publicados no período de 1963 a 2016.

Foram incluídos na pesquisa os pacientes com IRC que fazem hemodiálise no serviço de referência para tratamento hemodialítico $\square$ anteriormente Clínica de Doenças Renais $\square$ da $13^{a}$ Coordenadoria Regional de Saúde, localizado no município de Santa Cruz do Sul. Foram incluídos adultos maiores de 18 anos de idade que concordaram em participar da pesquisa e assinaram o termo de consentimento livre e esclarecido, totalizando 76 pacientes. Foram excluídos aqueles pacientes que não assinaram o termo ou que retiraram seu consentimento, a qualquer momento, durante a pesquisa e os pacientes debilitados, indispostos ou internados em unidades de terapia intensiva.

O estudo foi aprovado pelo Comitê de Ética em Pesquisa com Seres Humanos da Universidade de Santa Cruz do Sul (Parecer no 1.557.915). Cada participante recebeu um termo de consentimento livre e esclarecido em duas vias, em que uma via ficou com o participante, e a outra, com os pesquisadores.

Os pacientes foram selecionados com a ajuda da enfermeira responsável pela instituição, que excluiu os pacientes que estavam debilitados. Os dados foram coletados nos meses de julho e agosto de 2016.

A coleta de dados foi em forma de questionário (Apêndice A). Após preencher o questionário, o paciente passou por exame clínico de índice de placa visível (IPV) e de índice de sangramento gengival (ISG) (Apêndice B), os quais são de suma importância para a determinação do perfil de higiene bucal, revelando o hábito de higienize bucal, se é realizado com sucesso, segundo Löe e Silness ${ }^{9}$ (1963).

Como limitações do método utilizado, pode-se citar a influência da xerostomia e das medicações administradas aos pacientes da pesquisa, abordadas na discussão deste trabalho $\mathrm{O}$ exame clínico foi realizado em poltronas reclináveis, seguindo as normas de biossegurança tanto no que se refere ao examinador como aos instrumentos por ele utilizados; e os aparelhos utilizados foram: espelho clínico intrabucal número 5 (Duflex ${ }^{\circledR}$, Rio de Janeiro, Brasil), sonda periodontal milimetrada do tipo Williams (Millennium® ${ }^{\circledR}$, São Caetano do Sul, São Paulo, Brasil), pinça para algodão (Duflex®, Rio de Janeiro, Brasil) e roletes de algodão (Apolo®, Cataguases, Minas Gerais, Brasil).

A examinadora foi calibrada com auxílio do professor orientador do projeto. Sobre os resultados da calibragem, a concordância intraexaminadora foi mensurada pelo cálculo Kappa, indicador que elimina a possibilidade de os resultados coincidirem por acaso. O cálculo do IPV teve concordância excelente $(0,999518)$, e o cálculo do ISG teve concordância perfeita $(1,00000){ }^{8}$

Os resultados do estudo foram analisados por estatística descritiva e testes de hipóteses. $\mathrm{Na}$ etapa dos testes estatísticos, foram utilizados a ANOVA (Análise de Variância) e o Teste t de Student, afim de se verificar as diferenças entre médias de variáveis quantitativas. Para avaliar as associações entre variáveis categóricas, foi utilizado o Teste Qui-Quadrado de independência. O nível de significância utilizado como critério de aceitação ou rejeição nos testes estatísticos foi de $5 \%(\mathrm{p}<0,05)$. As análises foram realizadas no programa Statistical Package Social Sciences, versão 24.

\section{Resultados}

A pesquisa foi dividida em duas etapas e envolveu 76 pacientes com idades entre 22 e 89 anos (Tabela 1 ), em que $59,21 \%$ são do gênero masculino, representando 45 pacientes.

Tabela 1 - Faixa etária

\begin{tabular}{c|c|c|c|c|c}
\hline \multicolumn{2}{c|}{ Idade } & $\begin{array}{c}\text { Frequên- } \\
\text { cia }\end{array}$ & $\begin{array}{c}\text { Porcen- } \\
\text { tagem }\end{array}$ & $\begin{array}{c}\text { Porcen- } \\
\text { tagem } \\
\text { válida }\end{array}$ & $\begin{array}{c}\text { Porcen- } \\
\text { tagem } \\
\text { cumu- } \\
\text { lativa }\end{array}$ \\
\hline \multirow{5}{*}{ Válido } & De 22 a 39 anos & 4 & 5,3 & 5,3 & 5,3 \\
& De 40 a 49 anos & 8 & 10,5 & 10,5 & 15,8 \\
& De 50 a 59 anos & 15 & 19,7 & 19,7 & 35,5 \\
& De 60 a 69 anos & 27 & 35,5 & 35,5 & 71,1 \\
& De 70 a 89 anos & 22 & 28,9 & 28,9 & 100,0 \\
& Total & 76 & 100,0 & 100,0 & \\
\hline
\end{tabular}

Fonte: dados da pesquisa. 
Dos pacientes que utilizam prótese total (39,47\%), 30\% (9 pacientes) possuem somente prótese total superior. Entre os motivos para a perda dos dentes, prevaleceu a cárie dentária (63 pacientes - $82,89 \%$ da população estudada), seguida da mobilidade por doença periodontal (10 pacientes $13,15 \%$ ) e por trauma ( 3 pacientes $-3,94 \%$ ).

Sobre os hábitos de higiene bucal, 26 pacientes $(56,5 \%)$ relataram escovar os dentes três vezes ao dia, enquanto 6 pacientes (13\%) afirmaram escovar somente uma vez ao dia. Dos 46 pacientes dentados, somente $5(10,9 \%)$ já notaram haver sangramento gengival durante a escovação. Quanto à utilização do fio dental, 11 pacientes $(23,9 \%)$ responderam positivamente, entre eles, 7 pacientes $(63,6 \%)$ declararam utilizar com frequência de uma vez ao dia.

Apenas 11 pacientes $(23,9 \%)$ declararam realizar bochechos diários com alguma solução, e 22 pacientes $(47,8 \%)$, afirmaram sentir gosto ruim na boca ou ter mau hálito. Em relação às visitas ao dentista, somente 25 pacientes $(54,3 \%)$ afirmaram ir ao cirurgião-dentista regularmente. Quanto à frequência, foram obtidos os seguintes resultados (Tabela 2):

Tabela 2 - Frequência de idas ao cirurgião-dentista

\begin{tabular}{|c|c|c|c|c|c|}
\hline & & & & & \\
\hline & & Frequência & $\begin{array}{c}\text { Porcen- } \\
\text { tagem }\end{array}$ & $\begin{array}{c}\text { Porcen- } \\
\text { tagem } \\
\text { válida }\end{array}$ & $\begin{array}{c}\text { Porcen- } \\
\text { tagem } \\
\text { cumulativa }\end{array}$ \\
\hline \multirow{4}{*}{ Válido } & A cada 6 meses & 13 & 28,3 & 39,4 & 39,4 \\
\hline & Quando há dor & 11 & 23,9 & 33,3 & 72,7 \\
\hline & Outros & 9 & 19,6 & 27,3 & 100,0 \\
\hline & Total & 33 & 71,7 & 100,0 & \\
\hline Omisso & 0 & 13 & 28,3 & & \\
\hline \multicolumn{2}{|r|}{ Total } & 46 & 100,0 & & \\
\hline
\end{tabular}

Fonte: dados da pesquisa.

Dos 9 pacientes $(19,6 \%)$ que responderam a alternativa "outros", $4(44,44 \%)$ relataram ir ao dentista uma vez a cada ano, $2(22,22 \%)$ afirmaram ir a cada 2 anos, $2(22,22 \%)$ mencionaram que foram ao cirurgião-dentista somente antes de entrar na fila de transplante, e 1 paciente $(11,12 \%)$ afirmou ir todo o mês, pois utiliza aparelho ortodôntico.

Perguntas sobre a história médica dos pacientes também foram realizadas, e, posteriormente, as respostas foram verificadas com o histórico digital da instituição. Em relação às doenças sistêmicas que levaram o paciente à IRC, estão: hipertensão (70 pacientes - 92,10\%), diabetes (31 pacientes - 40,78\%), câncer renal (4 pacientes - 5,26\%), glomerulonefrite membranosa (5 pacientes $-6,57 \%$ ) e litíase de repetição (10 pacientes $-13,15 \%)$.

Dos 76 pacientes, cinco $(6,57 \%)$ já foram transplantados e estão novamente em terapia substitutiva. Quanto ao tempo em hemodiálise, foram obtidos os seguintes resultados, apresentados na Tabela 3:
Tabela 3 - Tempo de hemodiálise

\begin{tabular}{|c|c|c|c|c|c|}
\hline & & $\begin{array}{c}\text { Frequên- } \\
\text { cia }\end{array}$ & $\begin{array}{l}\text { Porcen- } \\
\text { tagem }\end{array}$ & $\begin{array}{c}\text { Porcen- } \\
\text { tagem } \\
\text { válida }\end{array}$ & $\begin{array}{c}\text { Porcen- } \\
\text { tagem } \\
\text { cumu- } \\
\text { lativa }\end{array}$ \\
\hline \multirow{6}{*}{ Válido } & $<1$ ano & 12 & 15,8 & 15,8 & 15,8 \\
\hline & De 1 a 4 anos e 11 meses & 30 & 39,5 & 39,5 & 55,3 \\
\hline & De 5 a 9 anos e 11 meses & 23 & 30,3 & 30,3 & 85,5 \\
\hline & De 10 a 15 anos e 11 meses & 9 & 11,8 & 11,8 & 97,4 \\
\hline & De 16 a 30 anos & 2 & 2,6 & 2,6 & 100,0 \\
\hline & Total & 76 & 100,0 & 100,0 & \\
\hline
\end{tabular}

Fonte: dados da pesquisa.

Dos pacientes que ainda possuem dentes naturais, somente um $(2,17 \%)$ não apresentou perdas dentárias, e a prevalência para as perdas foi a cárie $(84 \%)$. Sobre a promoção de saúde bucal dentro da instituição, 14 pacientes $(18,2 \%)$ afirmaram que tiveram orientação de higiene bucal uma vez enquanto realizavam a sessão de hemodiálise. Quanto à autoavaliação das condições de saúde bucal dos pacientes que possuem dentes naturais, foram obtidos os seguintes resultados (Tabela 4):

Tabela 4 - Autoavaliação da saúde bucal dos pacientes com dentes naturais

\begin{tabular}{c|c|c|c|c|c}
\cline { 3 - 6 } \multicolumn{1}{c|}{} & Frequência & Porcentagem & $\begin{array}{c}\text { Porcentagem } \\
\text { válida }\end{array}$ & $\begin{array}{c}\text { Porcentagem } \\
\text { cumulativa }\end{array}$ \\
\hline \multirow{4}{*}{ Válido } & Ótima & 2 & 4,3 & 4,3 & 4,3 \\
\cline { 3 - 6 } & Boa & 18 & 39,1 & 39,1 & 43,5 \\
& Regular & 17 & 37,0 & 37,0 & 80,4 \\
& Ruim & 7 & 15,2 & 15,2 & 95,7 \\
& Péssima & 2 & 4,3 & 4,3 & 100,0 \\
\cline { 3 - 6 } & Total & 46 & 100,0 & 100,0 & \\
\hline
\end{tabular}

Fonte: dados da pesquisa.

Acerca dos exames clínicos, 8 pacientes $(17,4 \%)$ apresentaram mobilidade dentária por doença periodontal severa. Houve alterações estomatológicas: 4 pacientes $(5,26 \%)$ tiveram resultado positivo, apresentando herpes simples recorrente $(1,31 \%)$, aftas na língua $(2,63 \%)$ e hiperceratose na mucosa jugal $(1,31 \%)$.

Resultados importantes foram a alta prevalência de presença de placa na maioria dos pacientes (Figura 1) e de ISG (Figura 2), com resultado significativamente baixo em relação ao IPV, o que é discutido a seguir. 
Figura 1 - Faixas do índice de placa visível

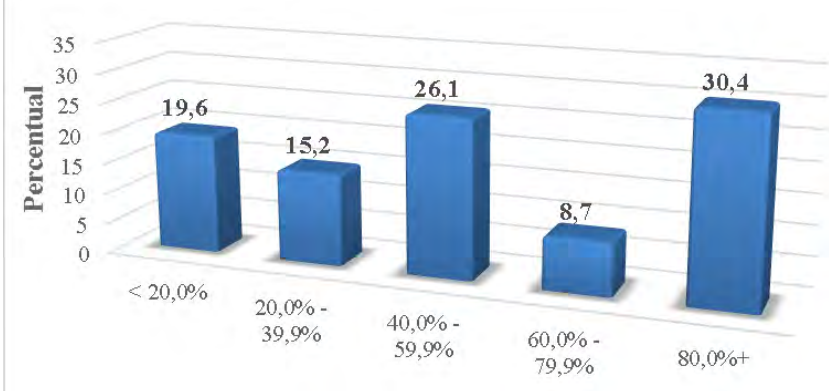

Fonte: dados da pesquisa.

Figura 2 - Faixas do índice de sangramento gengival

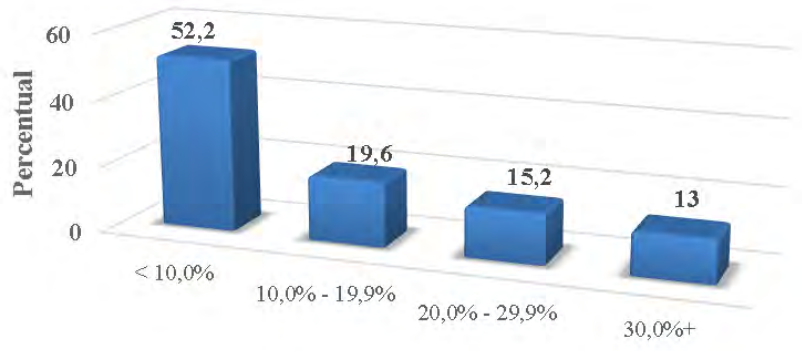

Fonte: dados da pesquisa.

Dados encontrados no histórico médico da instituição revelaram que 10 dos pacientes $(13,15 \%)$ que participaram da pesquisa não estão na fila do transplante. As medicações mais utilizadas são: eritropoetina (63 pacientes - 82,9\%), hidróxido de ferro $(81,6 \%)$, ácido fólico $(89,5 \%)$, complexo B $(88,2 \%)$ e carbonato de cálcio $(67,1 \%)$. Ainda utilizam enalapril $(21,1 \%)$ e sertralina $(7,9 \%)$.

Discussão

A inter-relação entre o perfil de higiene bucal e a condição sistêmica dos pacientes que frequentam clínicas de terapia renal substitutiva não é um tema discutido dentro dos centros de hemodiálise. Atualmente, novas pesquisas mostram que há necessidade de inclusão do cirurgião-dentista no tratamento interdisciplinar, para melhor qualidade de vida desses pacientes..$^{2,5,6,10-14}$ Sendo assim, esta pesquisa buscou contribuir com os estudos preexistentes, enriquecendo a literatura nessa área que ainda necessita ser melhor compreendida.

Foram avaliados 76 pacientes, com faixa etária de 22 a 89 anos, dos quais 59,21\% são do gênero masculino, e 39,47\% (30 pacientes) utilizam prótese total. A média do IPV foi $54,137 \%$, e do ISG, $16,765 \%$. As principais doenças que levaram os pacientes à IRC foram: hipertensão $(92,10 \%)$, diabetes $(40,78 \%)$, litíase por repetição $(13,15 \%)$, glomerulonefrite membranosa $(6,57 \%)$ e câncer renal $(5,26 \%)$. Esses resultados concordaram com os estudos previamente realizados. ${ }^{1,5,12}$

Ao analisar o percentual de pacientes que possuem prótese, o número de indivíduos que utilizam somente prótese total superior é significativamente grande $(30 \%)$. Eles apresentam dificuldade para se alimentar, o que é um fator prejudicial para a qualidade de vida, segundo a nutricionista da instituição.

Estudo realizado por Lacerda et al. ${ }^{10}$ (2015) comprovou que os pacientes edêntulos têm melhor saúde bucal, quando se trata de aptidão para transplante, devido a baixos níveis de infecções oportunistas bucais. $\mathrm{O}$ mesmo estudo constatou ser maior o índice de cáries em pacientes que estão há mais tempo em hemodiálise e não tiveram orientação de higiene bucal durante esse tempo.

A partir dos resultados desta pesquisa, observou-se prevalência da perda de dentes por cárie $(82,89 \%)$. Sabendo que a presença de cárie, lesões endodônticas e periodontites é a porta de entrada para microrganismos patógenos que se estabelecem na corrente sanguínea, essas condições podem levar ao aumento da mortalidade dos pacientes renais crônicos, principalmente do pacientes em terapia imunossupressora pós-transplante, pois tais patógenos tornam-se mais agressivos ainda, podendo lesar o órgão transplantado. ${ }^{13,15,16}$ Dos participantes desta pesquisa, cinco tiverem rejeição do transplante por infecções e nefropatia crônica do enxerto, o que os levou à hemodiálise novamente.

Outro fator que deve ser discutido é a redução do fluxo salivar integral, que aumenta a suscetibilidade a cárie e destruição dental. Isso ocorre devido à incapacidade de remoção do biofilme acumulado na superfície dental que, unido a má higiene bucal, resulta em processo carioso. ${ }^{13}$

Pesquisa publicada em $2007^{16}$ revelou que o fluxo salivar dos pacientes em hemodiálise é, em média, 0,60 $\mathrm{ml} / \mathrm{min}$, o que caracteriza hiposalivação, devido à restrição da ingestão de líquidos e à administração de medicamentos. Várias são as medicações utilizadas por pacientes em hemodiálise que causam a hipossalivação. A população participante desta pesquisa utiliza principalmente antidepressivos, anti-hipertensivos, analgésicos, relaxantes musculares e diuréticos. ${ }^{17}$

Existem literaturas que evidenciam a administração da eritropoetina por pacientes renais crônicos devido à presença de anemia normocítica, em que ocorre menor produção de células vermelhas, por haver deficiência do hormônio eritropoietina. ${ }^{10,18}$ Nesta pesquisa, $82,9 \%$ dos pacientes do serviço de referência para tratamento hemodialítico utilizam essa medicação, sendo a palidez na mucosa bucal característica da anemia que pode ser notada na maioria dos pacientes examinados. ${ }^{14,18}$ A redução da proteína $\mathrm{C}$ reativa ocorre quando há um tratamento da doença periodontal em pacientes renais crônicos, o que, unido à adequada condição nutricional, melhora a resposta à eritropoietina. ${ }^{19}$

Os estudos encontrados confirmam que pacientes renais crônicos apresentam elevado acúmulo de placa e de cálculo dentário, o que pode ser justificado 
pela menor produção salivar e pela suplementação de cálcio e fósforo usada por esses pacientes. ${ }^{2,12,13,16}$

Os níveis elevados de ureia aumentam o $\mathrm{pH}$ salivar, pois a ureia salivar é degradada em amônia, levando à alcalinização bucal; com isso, ocorre maior formação de cálculo dental. ${ }^{12,17}$ Tal evidência também foi significativa nesta pesquisa, já que $30,4 \%$ dos pacientes apresentaram $80 \%$ ou mais faces com presença de cálculo dental.

A presença de sangramento gengival não teve influência nas variáveis analisadas e não justifica a grande presença de placa e cálculo avaliados nesta pesquisa. $\mathrm{O}$ que pode ser relevante para o entendimento deste fato é que os pacientes em hemodiálise apresentam reduzida resposta inflamatória, quando comparados a pacientes saudáveis. ${ }^{2}$ Outros estudos evidenciam que a gengiva marginal se apresenta frequentemente inflamada, com ocorrências de hemorragias, fato que contrapõe os presentes resultados..$^{16,17}$

A diminuição do fluido gengival crevicular (FGC) possivelmente tem influência sobre essa resposta inflamatória gengival deficiente, visto que o FGC (fosfatase alcalina, $\square$-glucoronidase, aspartato aminotrans-ferase, prostaglandinas, imunoglobulinas G4, inter leucina-1) está presente em maior quantidade em sítios de agressão tecidual periodontal. Na hipossalivação, a redução no meio bucal e no sulco gengival pode levar à menor resposta às agressões, resultando em baixos ISGs. ${ }^{20}$

O tempo a que os pacientes são submetidos à hemodiálise, relacionado ao perfil de higiene bucal, tem grande relevância, o que é comprovado nos dados desta pesquisa e em pesquisas anteriores. Pois, com o passar dos anos, há redução da capacidade funcional, diminuição da autoestima, aumento da idade e aparição de outras complicações clínicas, aumentando o tempo de hemodiálise. Quanto maior o período, em anos, que o paciente passa dentro dos centros de hemodiálise, menos ele procura ações de prevenção e de terapêutica em saúde bucal, diminuindo a qualidade de vida. ${ }^{10,11,21}$

Somente 25 pacientes relataram frequentar o dentista, mesmo que com frequência reduzida. Entre estes, estão os pacientes mais jovens e em hemodiálise a menos tempo. A maioria dos pacientes com idade mais avançada utiliza prótese total ou tem necessidade de utilizá-la.

Segundo os resultados encontrados na população avaliada, 13,15\% da população não está apta a realizar o transplante renal, por apresentar idade avançada ou condição cardiovascular comprometida. Alguns desses pacientes não aptos procuraram serviço de assistência odontológica privada ou pública, mas, por apresentarem cardiopatias e diabetes descompensada, não tiveram condições ou liberação médica para realizar o procedimento de sua necessidade, fato que foi discutido também no estudo de Almeida et al. ${ }^{5}$ (2011).
Todo encaminhamento para realização de transplante é definido conforme o centro transplantador. Logo, o encaminhamento ao dentista previamente à recepção de um rim, como condição para que a cirurgia possa ser realizada, não é universal..$^{22}$ Com isso, muitos centros de diálise não preconizam a promoção da saúde bucal dos seus pacientes, para que, futuramente, possam entrar na fila para transplante.

No entanto, estudos revelam que a doença periodontal (decorrente dos hábitos de higiene bucal, evoluindo para inflamações gengival e periodontal) pode levar à deterioração mais rápida da função renal dos transplantes, já que, após passar pela cirurgia, os pacientes têm imunossupressão, período em que qualquer infecção pode ser agravada, tornando-se muitas vezes fatal. ${ }^{14,23,24}$

A Agência Nacional de Vigilância Sanitária é responsável pelo regulamento que possibilita o funcionamento dos serviços de diálise. Em sua última sessão sobre os cuidados com pacientes renais crônicos, definiu a obrigatoriedade de esses serviços possuírem uma equipe formada de médico, enfermeiro e técnico em enfermagem, psicólogo, nutricionista e assistente social. ${ }^{25}$

A saber, após todas as evidências relevadas nos estudos discutidos e com a comprovação da importância da presença do cirurgião-dentista em centros de hemodiálise, questiona-se a possibilidade de $o$ Ministério da Saúde repensar a equipe de atenção aos pacientes com doença renal crônica.

\section{Conclusão}

Apesar de a maioria dos pacientes $(80,43 \%)$ autoavaliarem sua saúde bucal de ótima a regular, salienta-se o perfil de higiene bucal precário, com alto índice de placa e cálculo dental, o que pode ser a causa da grande prevalência de perdas dentárias $(98,68 \%)$. Quanto à frequência dos pacientes que vão ao cirurgião-dentista ou à atenção odontológica que tiveram durante o tempo que estão em hemodiálise, foi encontrado um índice muito baixo, quando comparado à importância da saúde bucal frente à saúde geral e à qualidade de vida.

Conclui-se que os pacientes renais crônicos avaliados necessitam de atenção odontológica diversificada e integral. Com isso, medidas devem ser tomadas para a melhoria da atenção à saúde desses pacientes, facilitando distribuição da informação, avaliação e atenção periódica.

Por conseguinte, a inserção do cirurgião-dentista em centros de hemodiálise é de extrema importância para a prevenção e a promoção em saúde bucal dos pacientes em hemodiálise, resultando em maior possibilidade de sucesso nos casos de transplantes renais. 


\section{Abstract}

Introduction: oral hygiene practices of hemodialysis patients could turn better their health care and enable a successful kidney transplantation. However, this is not spoken subject in substitutionary therapy (hemodialysis) nowadays. Objectives: evaluate kidney chronic patients oral hygiene in their hemodialysis treatment. Materials and methods: this is an analytic transversal observational study, which population were patients with chronic kidney disease, at the current service for hemodialysis treatment, at the 13 $\square$ Health Sectional Coordination Body, placed at Santa Cruz do Sul city, RS. The used technique to data gathering was interpersonal interview standardized and intraoral clinical exams, along with medical digital historic of the institution. Results: the sample was male predominance $(59,21 \%)$, age between 22-89 years old. Among 76 research patients, 39,47\% have full prosthesis, IPV average's prevalence was $54,137 \%$, and ISG's $16,765 \%$. Study's results were analyzed among descriptive statistics and hypothesis tests. The meaningfulness level used as acceptance or rejection criterion on the statistical tests was $5 \%(p<0,05)$. Conclusion: evaluated hemodialysis patients have a precarious oral hygiene profile combined with high plaque prevalence and dental calculus, which proves the necessity of integral and diversity dental assistant to endure awareness and buccal health.

Keywords: Chronic diseased odontology assistant. Buccal hygiene avaliation. Chronic kidney insufficiency.

\section{Referências}

1. 1.Sesso RC, Lopes AA, Thomé FS, Lugon JR, Martins CT Inquérito Brasileiro de Diálise Crônica 2016. J Bras Nefrol 2017; 39(3):262-6.

2. 2.Souza CRD, Libério AS, Guerra RNM, Monteiro S, Silveira EJD, Pereira ALA. Avaliação da condição periodontal de pacientes renais em hemodiálise. Rev Assoc Med Bras 2005; 51(5):285-9.

3. 3.Bhatsange A, Patil SR. Assessment of periodontal health status in patients undergoing renal dialysis: A descriptive, cross-sectional study. J Indian Soc Periodontol 2012; 16(1):37-42.

4. 4.Bayraktar G, Kurtulus I, Duraduryan A, Cintan S, Kazancioglu R, Yildiz A. Effect of educational level on oral health in peritoneal and hemodialysis patients. Int J Dent 2009; (3):1-

5. 5.Almeida DC, Pereira CS, Granjeiro JM, Machado WAS, Tostes FRV, Barboza ESP, et al. Relação bidirecional entre doença periodontal e doença renal crônica: da progressão da doença renal crônica à terapia renal substitutiva de diálise. Rev de Period 2011; 21(1):73-9.

6. 6.Barros DCP, Cordova LHS, Velandia AAL, Souza DM. Avaliação dos Hábitos de Higiene Bucal de Pacientes em Hemodiálise do Hospital Regional do Vale do Paraíba. Braz J Periodontol 2014; 24(3):7-18.

7. 7.Guevara HG, Mónaco GL, Riveiro CS, Vasconcellos V, Souza DP, Raitz R. Manejo odontológico em pacientes com doença renal crônica. Rev Bras de Ciênc da Saúde 2014; 12(40):74-81.

8. 8.Susin C, Rösing CK. Praticando odontologia baseada em evidências. 2. ed. Canoas: Ed. da ULBRA; 2002.

9. 9.Löe H, Silness J. Periodontal Disease in Pregnancy I. Prevalence and Severity. Acta Odont Scand 1963; 21(6):533-51.
10. 10.Lacerda MCSR, Viana KB, Dores DF, Bessa-Nogueira RV, Ribeiro CMB. Caracterização da saúde bucal de indivíduos renais crônicos aptos a transplante. Rev Odontol UNESP $2015 ;$ 44(5):292-8.

11. 11.Jain S, Singla A, Basavaraj P, Singh S, Singh K, Kundu $\mathrm{H}$. Underlying kidney disease and duration of hemodialysis: an assessment of its effect on oral health. J Clin Diagn Res $2014 ; 8(5): 65-9$.

12. 12.Rossi SS, Glick M. Dental considerations for the patient with renal disease receiving hemodialysis. J Am Dent Assoc 1996; 127(2):211-9.

13. 13.Fujimaki M, Rosa OPS, Torres SA. Microrganismos cariogênicos em pacientes com insuficiência renal crônica em hemodiálise. Rev Odontol Univ São Paulo 1998; 12(2):149-58.

14. 14.Filho JZC, Padilha WSM, Santos EKN. Cuidados odontológicos em portadores de insuficiência renal crônica. Rev Cir Traumat Buco-maxilo-fac 2007; 7(2):19-28.

15. 15.Bots CP, Brand HS, Veerman EC, Valentijn-Benz M, Van Amerongen BM, Valentijn RM, et al. Interdialytic weight gain in patients on hemodialysis is associated with dry mouth and thirst. Kidney Int 2004; 66(4):1662-8.

16. 16.Dias CRS, Sà TCV, Pereira ALA, Alves CMC. Avaliação da condição bucal em pacientes renais crônicos submetidos à hemodiálise. Rev Assoc Med Bras 2007; 56(6):510-4.

17. 17.Miguel LCM, Locks A, Neumann V. Redução do fluxo salivar em hemodialisados. J Bras Nefrol 2006; 28(1):20-4.

18. 18.Sonis ST, Fazio R, Fang LST. Princípios e prática de medicina oral. 2. ed. Rio de Janeiro: Guanabara Koogan; 1996.

19. 19.Siribamrungwong $M$, Yothasamutr K, Puangpanngam K. Periodontal treatment reduces chronic systemic inflammation in peritoneal dialysis patients. Ther Apher Dial 2014; 18(3):305-8.

20. 20.Chibebe PC, Terreri M, Ricardo LH, Pallos D. Uma visão atual do fluido gengival crevicular como método de diagnóstico periodontal. Rev Ciênc Med Campinas 2008; 17(3-6):16773.

21. 21.Guedes KD, Guedes HM. Qualidade de vida do paciente portador de insuficiência renal crônica. Ciênc Saúde 2012; $5(1): 48-53$

22. 22.Mantovani FF, Fregonesi A, Alves GF, Magna LA. Avaliação odontológica em pacientes diabéticos candidatos a transplante renal. Rev Assoc Paul Cir Dent 2009; 63(1):36-42.

23. 23.Ioannidou E, Shaqman M, Burleson J, Dongari-Bagtzoglou A. Periodontitis case definition affects the association with renal function in kidney transplant recipients. Oral Dis 2010; 16(7):636-42.

24. 24.Crawford JM. Periodontitis and cardiovascular disease. Dis Mon 2011; 57(4):203-5.

25. 25.Brasil. Ministério da Saúde. Resolução RDC no 389/2014, de 14 de marco 2014. Agência Nacional de Vigilância Sanitária. Estabelece o regulamento para o funcionamento dos serviços de diálise. Diário Oficial da União, Brasília, 14 mar 2014, seção 1, p. 35.

\section{Endereço para correspondência:}

Kellyn de Bastos Silva

Rua Itaqui, 404, Arroio Grande

96835-160, Santa Cruz do Sul, RS, Brasil

Telefone: (51) 99838-6604

E-mail: kellynbsilva@gmail.com

Recebido: 20/08/18. Aceito: 03/10/18. 